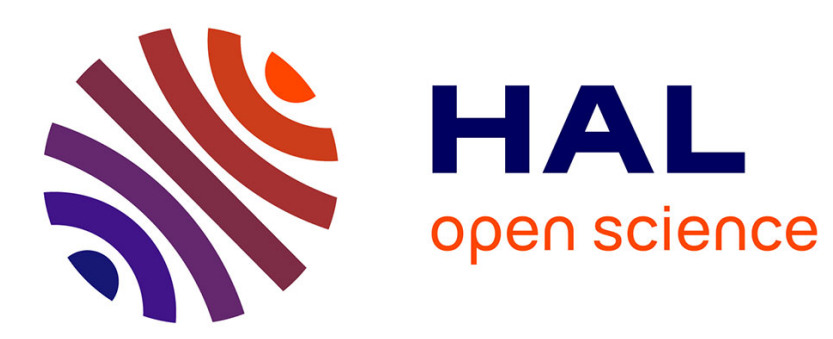

\title{
Segmentation of Bone Vessels in 3D Micro-CT Images Using the Monogenic Signal Phase and Watershed
}

\author{
Hao Xu, Max Langer, Françoise Peyrin
}

\section{To cite this version:}

Hao Xu, Max Langer, Françoise Peyrin. Segmentation of Bone Vessels in 3D Micro-CT Images Using the Monogenic Signal Phase and Watershed. 2020 IEEE 17th International Symposium on Biomedical Imaging (ISBI), IEEE, Apr 2020, Iowa City, United States. pp.741-744, 10.1109/ISBI45749.2020.9098530 . hal-03054374

\section{HAL Id: hal-03054374 \\ https://hal.science/hal-03054374}

Submitted on 11 Dec 2020

HAL is a multi-disciplinary open access archive for the deposit and dissemination of scientific research documents, whether they are published or not. The documents may come from teaching and research institutions in France or abroad, or from public or private research centers.
L'archive ouverte pluridisciplinaire HAL, est destinée au dépôt et à la diffusion de documents scientifiques de niveau recherche, publiés ou non, émanant des établissements d'enseignement et de recherche français ou étrangers, des laboratoires publics ou privés. 


\title{
SEGMENTATION OF BONE VESSELS IN 3D MICRO-CT IMAGES USING THE MONOGENIC SIGNAL PHASE AND WATERSHED
}

\author{
Hao Xu, Max Langer, Françoise Peyrin \\ Univ Lyon, CNRS UMR 5220, Inserm U1206, INSA Lyon, Université Claude Bernard Lyon 1, Creatis,
F69621 Villeurbanne Cedex, France.
}

\begin{abstract}
We propose an algorithm based on marker-controlled watershed and the monogenic signal phase asymmetry for the segmentation of bone and micro-vessels in mouse bone. The images are acquired using synchrotron radiation micro-computed tomography $(\mathrm{SR}-\mu \mathrm{CT})$. The marker image is generated with hysteresis thresholding and morphological filters. The control surface is generated using the phase asymmetry of the monogenic signal in order to detect edgelike structures only, as well as improving detection in low contrast areas, such as bone-vessel interfaces. The quality of segmentation is evaluated by comparing to manually segmented images using the Dice coefficient. The proposed method shows substantial improvement compared to a previously proposed method based on hysteresis thresholding, as well as compared to using the gradient image as control surface. The algorithm was applied to images of healthy and metastatic bone, permitting quantification of both bone and vessel structures.
\end{abstract}

Index Terms - synchrotron radiation, micro-tomography, watershed, monogenic signal, vessel segmentation.

\section{INTRODUCTION}

Breast cancer is one of the most common cancer in women, with approximately 508,000 deaths annually worldwide [1][2]. Around $70 \%$ of patients with breast cancer develop bone metastases, leading to osteolytic and osteoblastic lesions [3]. Bone metastasis is currently incurable, and median overall survival times have been reported in the range of 24-65 months [4]-[6]. The vessel networks in bone is thought to play an important role in cancer and metastasis growth. In breast cancer bone metastasis, there is an abnormal growth of blood vessels in the bone [7].

Previously, three-dimensional (3D) X-ray $\mu \mathrm{CT}$ with a contrast agent has been used to visualize the bone vascularization [8]. This required demineralization of the bone however, precluding simultaneous quantitative analysis of bone and vessels.

SR X-ray phase contrast CT has been used for the simultaneous visualization of calcified bone tissue and vasculature in C57BL/6 mice post mortem without any staining or contrast agent [9]. However, due to the high resolution necessary for this imaging protocol, the field of view is limited. Further, the phase contrast in vessels was relatively weak, so that vessels had to be segmented manually, making the applicability to large data sets limited.

$\mathrm{X}$-ray $\mu \mathrm{CT}$ has been used to examine alterations in cortical bone micro-porosity, mineralization, and cancellous bone architecture due to estrogen deficiency in an ovariectomized rat model of postmenopausal osteoporosis [10]. In this study, the vessels were not directly imaged, but only cortical vascular canals.

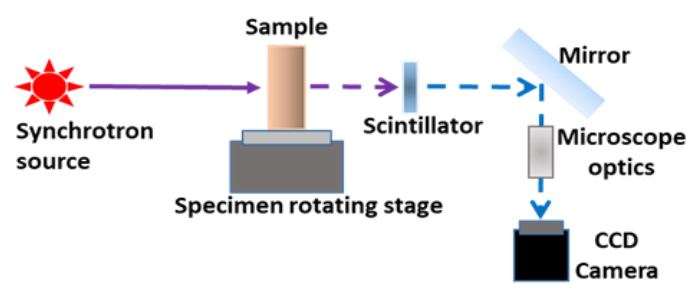

Fig. 1. Schematic of the imaging setup.

We previously developed a protocol to simultaneously analyze in 3D the micro-vascularization and bone structure in rat bone using SR- $\mu$ CT images [11]. This protocol was based on hysteresis thresholding and morphological filtering, and permitted the quantification of large data sets.

The aim of this work is to quantify bone and vascularization in mouse bone based on SR- $\mu$ CT images. Transition from rats to mice makes models of many pathologies available, but presents a number of challenges. In mice bone, as opposed to rat, at the chosen imaging resolution ( $3.5 \mu \mathrm{m}$ voxel size), vessels appear to be in contact with the bone surface, precluding the use of hysteresis thresholding for the separation of bone and vessels. In addition, the contrast between bone and vessels contrast agent may be weaker.

To this aim, we present a 3D segmentation method based on the marker-controlled watershed algorithm [12] to separate bone and vessel compartments. An important step for this algorithm is the generation of markers. Here, we assume three segments: bone, vessels and background. Ideally, the marker image should contain no false positives, while including a marker in each connected component of each compartment. For this purpose, we used hysteresis thresholding and morphological filters. The other essential step is the generation of a control surface image from the image to be segmented, where the "flooding" of the watershed algorithm happens. Classically, the gradient of the original image is used. Due to the relatively low contrast between bone and vessel compared to vessel or bone to background, the gradient image does not yield a satisfying segmentation. Therefore, we propose to use the local phase asymmetry derived from the monogenic signal to generate the control surface, which quantifies whether the image is locally odd (edge-like) or even (line-like). This measure has the interesting property of invariance to signal intensity [13][14].

The algorithm was evaluated using manually segmented volumes extracted from the original data. Segmentation quality was measured using the Dice coefficient. The method was compared to our previous method based on hysteresis thresholding as well as marker-controlled watershed on the gradient. The proposed method shows substantial improvement. The algorithm was used to extract parameters to characterize bone and vessel structure in healthy and metastatic bone. 


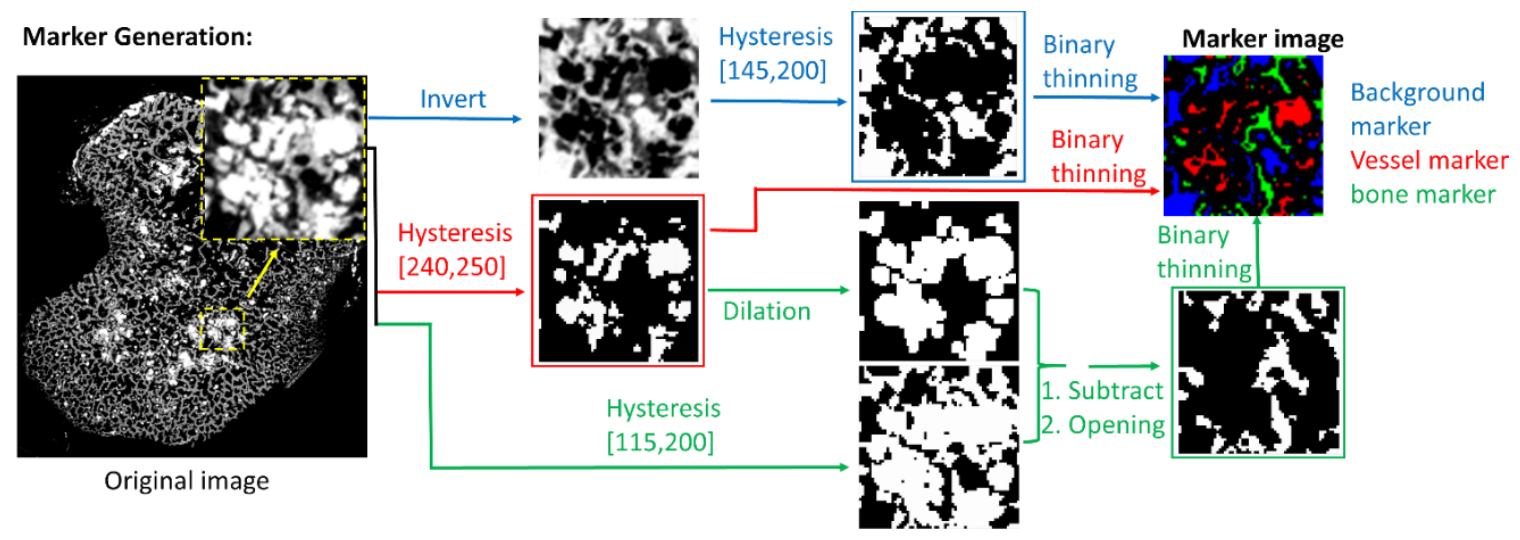

Fig. 2. Generation of the marker image for the marker-controlled watershed.

\section{MATERIALS AND METHODS}

\subsection{Sample preparation and image acquisition}

8 week-old female Balb/c nude mice were inoculated with luciferase-expressing human B02 breast cancer cells in the tail artery [15]. 22 days after the inoculation of tumor cells, the mice were sacrificed. The mice were perfused with contrast agent in the vascular system for imaging. We consider here two groups of samples: healthy and metastatic, with 5 mice per group.

Imaging was performed using $\mathrm{SR}-\mu \mathrm{CT}$ at the European Synchrotron Radiation Facility (ESRF) on the ID19 beamline. A sketch of the experimental setup is shown in Fig. 1. The X-ray energy was set to $26 \mathrm{keV}$ using the first harmonic of undulator radiation. Exposure time was set to $0.15 \mathrm{~s}$ per radiograph for approximately 8 minutes acquisition for one sample. 2000 radiographs of the sample were recorded using a $2048 \times 2048$ pixel CCD-based detector at evenly spaced angles of view over a $360^{\circ}$ rotation. This gave a field of view (FOV) as a cylinder of diameter $7 \mathrm{~mm}$. At the end, $2000 \times 2000 \times 1200$ voxels $3 \mathrm{D}$ images with an isotropic voxel size of $3.5 \mu \mathrm{m}$ were reconstructed from the projections using filtered back-projection.

\subsection{Marker based watershed}

The marker-controlled watershed algorithm permits to initialize the watershed from seeds in the image, which are known by some means [12]. This can reduce the number of iterations of the watershed algorithm, and simplifies the merging the resulting segments. The marker image should ideally contain at least one segment in each connected component of each class, while having a false positive rate close to zero. Here, we propose to use hysteresis thresholding and morphological thinning to generate the marker image.

Markers are generated for three segments in the image: bone, vessels and background. The procedure for the segmentation of the markers are given in Fig. 2. Background markers are given by blue arrows. Hysteresis thresholding is used to make an initial segmentation of the background. In order to ensure a low falsepositive rate, one iteration of morphological thinning is applied. Vessels markers are given by red arrows (Fig. 2). As with the background markers, an initial segmentation is performed using hysteresis thresholding which is followed by one iteration of morphological thinning. Bone markers are given by green arrows (Fig. 2). First, both bone and vessels are segmented together using hysteresis thresholding. Then, the vessel markers are subtracted and morphological opening is used to remove isolated small particles.
Finally, one iteration of morphological thinning is applied to minimize false positives. Background markers, vessel markers, and bone markers are shown in blue, red and green respectively in the final marker image (Fig. 2).

\subsection{Monogenic signal phase}

To steer the watershed algorithm, a control surface is generated from the original image. This can be the image itself, but is often based on the magnitude of the gradient. Here, the magnitude of the gradient does not perform satisfactory, due to the relatively low contrast at interfaces between bone and vessel, compared to vessel or bone to background. To alleviate this problem, we propose to use the local phase asymmetry of the 3D monogenic signal as the control surface for the watershed algorithm. The monogenic signal phase has the interesting property of being invariant to signal intensity: it only depends on the local shape of the signal. Here, we want to detect edge like structures only, so that a line yields two edge responses. For this purpose, we use the phase asymmetry measure, which quantifies whether the signal is locally odd (edge like) or even (line like).

The monogenic signal is the isotropic and multidimensional extension of 1-D analytic signal [14]. The 3D monogenic signal is formed by the combination of the original signal and and three components that are Riesz transforms of the original signal [16]:

$$
\begin{aligned}
& f_{m}(\boldsymbol{x})=\left(f(\boldsymbol{x}), R_{1} f(\boldsymbol{x}), R_{2} f(\boldsymbol{x}), R_{3} f(\boldsymbol{x})\right) \\
& \boldsymbol{R} f(\boldsymbol{x})=\left(R_{1} f(\boldsymbol{x}), R_{2} f(\boldsymbol{x}), R_{3} f(\boldsymbol{x})\right)
\end{aligned}
$$

where $f(\boldsymbol{x})$ is the original image, $\boldsymbol{R}$ is the Riesz transform and $\boldsymbol{x}=$ $\left(x_{1}, x_{2}, x_{3}\right) \in \mathbb{R}^{3}$ are the spatial coordinates. The Riesz operator component $R_{\alpha}: \mathbb{R}^{3} \mapsto \mathbb{R}$ is characterized by its frequency response

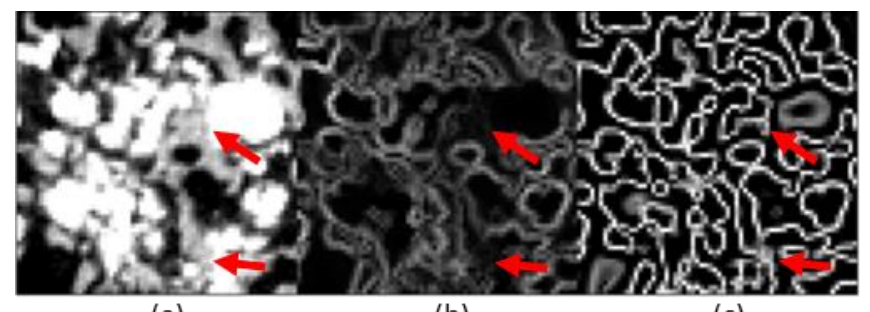

(a)

(b)

(c)

Fig. 3. Illustration of 3D edge detection. (a) zoom in an original gray level image, (b) gradient magnitude image. (c) multiscale 3D asymmetry computed from the monogenic signal; 


$$
\mathcal{F}\left[R_{\alpha} f\right](\boldsymbol{\omega})=-j \frac{\omega_{\alpha}}{\|\boldsymbol{\omega}\|} \hat{f}(\boldsymbol{\omega}), \alpha=1, \ldots, 3
$$

where $\boldsymbol{\omega}=\left(\omega_{1}, \omega_{2}, \omega_{3}\right) \in \mathbb{R}^{3}$ is the angular frequency variable conjugate to $\boldsymbol{x}$. $\alpha$ gives the 3D direction of the Riesz transformed components, corresponding to the directions of the basis vectors. $\mathcal{F}$ is the Fourier transform operator. Since structure may be scale dependent, features at a useful scale have to be isolated, usually by the use of bandpass filters [13]. Here we use log-Gabor filters. Thus, the $3 \mathrm{D}$ monogenic signal $f_{m g}(\boldsymbol{x})$ is constructed as:

$$
\begin{aligned}
& f_{m g}(\boldsymbol{x})= \\
& \left(f(\boldsymbol{x}) * g(\boldsymbol{x}), R_{1} f(\boldsymbol{x}) * g(\boldsymbol{x}), R_{2} f(\boldsymbol{x}) * g(\boldsymbol{x}), R_{3} f(\boldsymbol{x}) * g(\boldsymbol{x})\right)
\end{aligned}
$$

where $g(\boldsymbol{x})$ is the spatial domain representation of the log-Gabor filter, and $*$ denotes convolution operation. The 3D monogenic signal can also be expressed in terms of its even and odd components, which yields [17]:

$$
f_{m g}(\boldsymbol{x})=f_{m g e}(\boldsymbol{x})+i * f_{m g o}(\boldsymbol{x})
$$

where the even component is

$$
f_{m g e}(\boldsymbol{x})=f(\boldsymbol{x}) * g(\boldsymbol{x}),
$$

and the odd component

$$
f_{m g o}(\boldsymbol{x})=\left(\sum_{\alpha=1}^{3}\left|R_{\alpha} f(\boldsymbol{x}) * g(\boldsymbol{x})\right|^{2}\right)^{1 / 2}
$$

For the detection of edges in the image, we use the multiscale phase asymmetry measure, which is given by [13]:

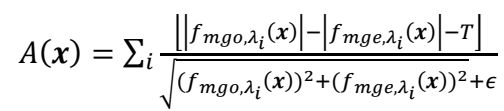

where $\mathrm{T}$ is a threshold for setting the sensitivity and suppressing noisy signals, $[$.$] is an operator which replaces negative values with$ zero, $\epsilon$ is small number to avoid division by zero, and $\left\{\lambda_{i}\right\}$ are a set of center-wavelengths $\left(\lambda_{i}=2 \pi / \omega_{i}\right)$ of the log-Gabor filters.

\section{RESULTS}

Qualitative results of the 3D edge detection are shown in Fig. 3: (a) shows the original image, which is a zoom on a $2 \mathrm{D}$ slice from the $3 \mathrm{D}$ volume. The red arrows show low contrast interfaces between bone and vessel. Fig. 3 (b) shows the magnitude of the gradient. The boundary between bone and vessel can not be detected due to the low contrast. Fig. 3 (c) shows the multiscale 3D phase asymmetry of the original image using $(\lambda=6,7,8)$. Lower scales contain too much edge details so that useful structures can not be recognized. Conversely, higher scales miss too many details. Edge detection at the low contrast interfaces between bone and vessel is substantially improved using the phase asymmetry, compared to the magnitude of the gradient. There are some spurious edges detected in homogeneous areas in the vessels. The contrast is relatively weak, however, and these edges, being inside solid areas, are always covered by a marker. Hence they do not influence the segmentation.

Fig. 4 illustrates the relationship between markers and control surfaces, and shows segmentation using hysteresis and watershed using a gradient based control surface. Fig. 4 (a) shows the original image, (b) shows the markers superposed on the gradient based control surface. Vessel markers are shown in red, bone markers in green, and background markers in blue. Fig. 4 (c) shows the markers
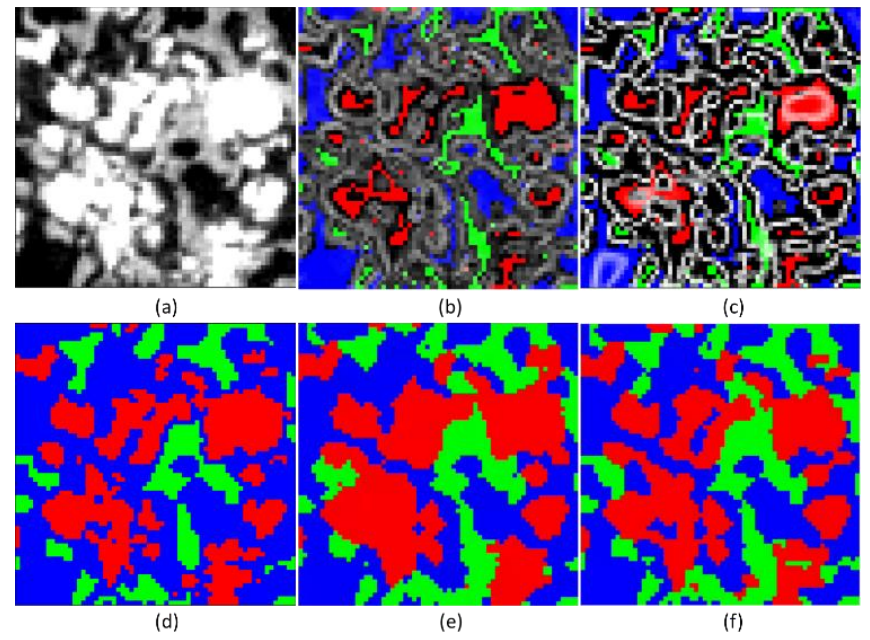

Fig. 4. Illustration of 3D segmentation results. (a) original image, (b) vessels marker (red), bone marker (green), background marker (blue) and the gradient based control surface. (c) markers and the monogenic signal phase based control surface. (d) segmentation result using hysteresis thresholding. (e) segmentation using gradient control surface based marker watershed. (f) segmentation with the proposed method using marker-controlled watershed and monogenic phase asymmetry.

overlayed on the monogenic phase asymmetry based control surface. Fig. 4 (d) shows the segmentation result using hysteresis thresholding, (e) shows the segmentation using the gradient based control surface watershed, and (f) shows the segmentation using the monogenic phase asymmetry based control surface watershed.

To validate the segmentation results, a representative volume (64×64×64 voxels) was manually segmented using VGStudio Max (Volume Graphics GmbH, Heidelberg, Germany).

Segmentation quality was measured using the Dice coefficient. The proposed method was compared to our previous protocol based on hysteresis thresholding as well as to marker-controlled watershed using a control surface based on the magnitude of the gradient. A comparison is shown in Tab. 1 . The proposed method substantially improved segmentation in terms of the Dice coefficient.

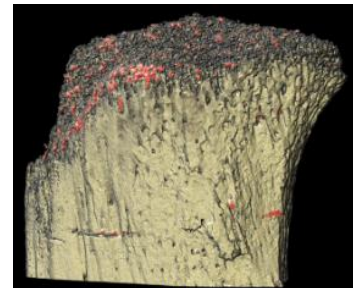

(a)

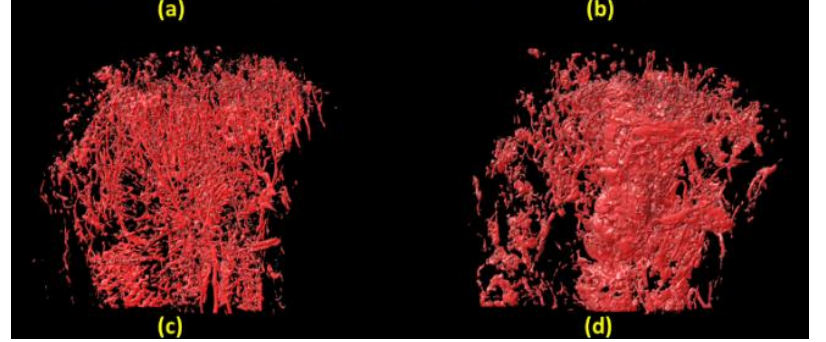

Fig. 5. 3D rendering volumes. (a) bone and vessel rendering in healthy group. (b) bone and vessel rendering in metastases group. (c) vessel rendering in healthy group. (d) vessel rendering in metastases group. 
TABLE 1. Dice coefficient for the different methods

\begin{tabular}{|c|c|c|c|}
\hline Dice & Vessel & Bone & Background \\
\hline $\begin{array}{c}\text { Hysteresis } \\
\text { thresholding }\end{array}$ & 0.8434 & 0.8055 & 0.8517 \\
\hline $\begin{array}{c}\text { Gradient }+ \\
\text { watershed }\end{array}$ & 0.9234 & 0.9127 & 0.9300 \\
\hline $\begin{array}{c}\text { Monogenic }+ \\
\text { watershed }\end{array}$ & 0.9695 & 0.9240 & 0.9711 \\
\hline
\end{tabular}

$3 \mathrm{D}$ renderings of a healthy sample and a sample with metastases segmented using the proposed method are shown in Fig. 5. The metastatic sample shows evident large bones lesions. Comparing (c) and (d), vessels in the metastatic sample appear thicker than in the healthy sample, which indicates an increased and abnormal vascularization, as expected.

Bone volume (BV) and vessel volume (VV) were measured by counting voxels in the corresponding compartments. Total volume (TV) of the samples was considered as the volume spanned by the outer contour of the bone. In order to compare the different samples, the normalized ratios: BV/TV, VV/TV were calculated. Mean vessel thickness (V.Th) was also extracted. Box plots of these parameters are shown in Fig. 6. Normal distribution of the parameters was verified using the Lilliefors test $(p>0.05)$. BV/TV was significantly lower in the metastatic group (ANOVA F-test, $\mathrm{p}<0.05$ ), which is consistent with the apparent large metastatic lesions visible in Fig. 5 (b). Although there was no significant difference in VV/TV ( $\mathrm{p}=$ $0.30)$ and V.Th $(p=0.09)$, possibly due to the limited sample size, the box plots in Fig. 6 still indicate that VV/TV and V.Th is increased in the metastatic bone, meaning there are both more and thicker vessels in the metastatic bone.

\section{CONCLUSION}

We presented an algorithm to segment bone and vessels in 3D SR$\mu \mathrm{CT}$ images of mouse bone with contrast agent, using the markercontrolled watershed on the monogenic signal phase asymmetry. The marker-controlled watershed permitted to address the problem of having vessels in contact to the bone surface, which precludes the correct use of hysteresis thresholding. In addition, the introduction of the monogenic signal phase asymmetry as control surface brought a substantial improvement of the segmentation of the relatively weakly contrasted bone and vessel interfaces. Our evaluation using manually segmented images showed substantial improvement in segmentation quality using the proposed method, compared to using hysteresis thresholding as well as using a gradient based control surface in the marker-controlled watershed. The algorithm was used to analyze healthy and metastatic bone. Several quantitative parameters were extracted to characterize bone and vessel structure. Statistical analysis revealed a decrease in bone volume, and indicated an increase in vessel volume and thickness in metastatic bone, as expected. The proposed protocol will be used to study the effects of anti-angiogenic drugs in bone metastasis.

\section{REFERENCES}

[1] C. Tulotta et al., "Endogenous Production of IL1B by Breast Cancer Cells Drives Metastasis and Colonization of the Bone Microenvironment," Clin. Cancer Res., vol. 25, no. 9, pp. 27692782, 2019.

[2] A. Coleman, M.P., Quaresma, M., Berrino, F., Lutz, J.M., De Angelis, R., Capocaccia, R., Baili, P., Rachet, B., Gatta, G.,
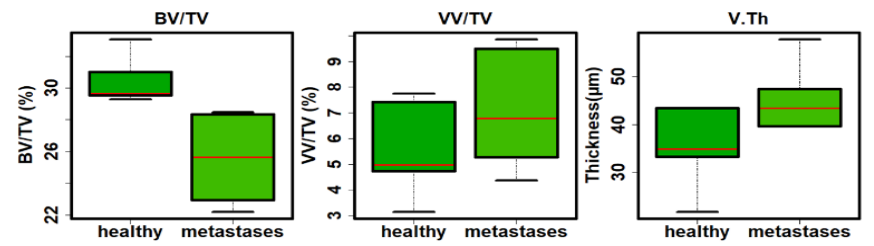

Fig. 6. Box plots of parameters extracted from the imaged mouse bone. Bone volume is decreased and vessel parameters increased in metastasis, as expected.

Hakulinen, T. and Micheli, "Cancer survival in five continents: a worldwide population-based study (CONCORD)," Lancet Oncol., vol. 9, no. 8, pp. 730-756, 2008.

[3] M. Akhtari et al., "Biology of breast cancer bone metastasis," Cancer Biol. Ther., vol. 7, no. 1, pp. 3-9, 2008.

[4] C. Pulido et al., "Bone metastasis risk factors in breast cancer," Ecancermedicalscience, vol. 11, no. 715, pp. 1-17, 2017.

[5] F. Nutter et al., "Different molecular profiles are associated with breast cancer cell homing compared with colonisation of bone : evidence using a novel bone-seeking cell line," Endocr. Relat. Cancer, vol. 21, no. 2, pp. 327-341, 2014.

[6] K. Lote, A. Walløe, and A. Bjersand, "Bone Metastasis Prognosis, Diagnosis and Treatment," Acta Radiol. Oncol., vol. 25 , no. 4-6, pp. 227-232, Jan. 1986.

[7] D. C. Herve Nyangoga, Philippe Mercier, Helene Libouban, Michel Felix Basle, "Three-Dimensional Characterization of the Vascular Bed in Bone Metastasis of the Rat by Microcomputed Tomography (MicroCT)," PLoS One, vol. 6, no. 3, pp. 1-8, 2011. [8] X. Zhang et al., "Periosteal Progenitor Cell Fate in Segmental Cortical Bone Graft Transplantations: Implications for Functional Tissue Engineering," J. BONE Miner. Res., vol. 20, no. 12, pp. 2124-2137, 2005.

[9] J. A. Núñez, A. Goring, E. Hesse, P. J. Thurner, P. Schneider, and C. E. Clarkin, "Simultaneous visualisation of calcified bone microstructure and intracortical vasculature using synchrotron Xray phase contrast-enhanced tomography," Sci. Rep., vol. 7, no. 1, p. $13289,2017$.

[10] D. Sharma et al., "The effects of estrogen deficiency on cortical bone microporosity and mineralization," Bone, vol. 110, pp. 1-10, 2018.

[11] M. Langer, R. Prisby, Z. Peter, A. Guignandon, M. H. LafageProust, and F. Peyrin, "Simultaneous 3D imaging of bone and vessel microstructure in a rat model," IEEE Trans. Nucl. Sci., vol. 58, no. 1, pp. 139-145, 2011.

[12] F. Meyer and S. Beucher, "Morphological segmentation," J. Vis. Commun. Image Represent., vol. 1, pp. 21-46, 1990.

[13] C. P. Bridge, "An Introduction To The Monogenic Signal," arXiv, vol. 1703, no. 09199, pp. 1-21, 2017.

[14] M. Felsberg and G. Sommer, "The monogenic signal," IEEE Trans. signal Process., vol. 49, no. 12, pp. 3136-3144, 2001.

[15] R. Bachelier, C. B. Confavreux, O. Peyruchaud, M. Croset, and D. Goehrig, "Combination of anti-angiogenic therapies reduces osteolysis and tumor burden in experimental breast cancer bone metastasis," Int. J. cancer, vol. 135, no. 6, pp. 1319-1329, 2014.

[16] N. Chenouard and M. Unser, "3D Steerable Wavelets in Practice," IEEE Trans. IMAGE Process., vol. 21, no. 11, pp. 4522 4533, 2012.

[17] J. A. N. Kashif Rajpoot, Vicente Grau, "Local phase based 3D boundary detection using monogenic signal and its application to real-time 3D echocardiography images," IEEE Int. Symp. Biomed. Imaging From Nano to Macro, pp. 783-786, 2009. 